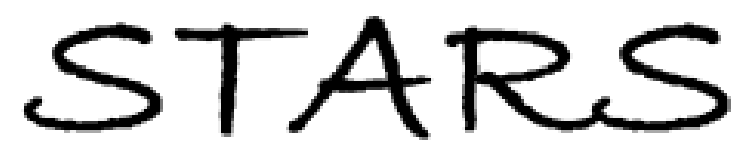

University of Central Florida

STARS

Faculty Bibliography 1990s

Faculty Bibliography

$1-1-1999$

\title{
Intensity dependent transmission dynamics in magnetic fluids
}

Tengda Du

University of Central Florida

Weili Luo

University of Central Florida

Find similar works at: https://stars.library.ucf.edu/facultybib1990

University of Central Florida Libraries http://library.ucf.edu

This Article; Proceedings Paper is brought to you for free and open access by the Faculty Bibliography at STARS. It has been accepted for inclusion in Faculty Bibliography 1990s by an authorized administrator of STARS. For more information, please contact STARS@ucf.edu.

\section{Recommended Citation}

Du, Tengda and Luo, Weili, "Intensity dependent transmission dynamics in magnetic fluids" (1999). Faculty Bibliography 1990s. 2608.

https://stars.library.ucf.edu/facultybib1990/2608

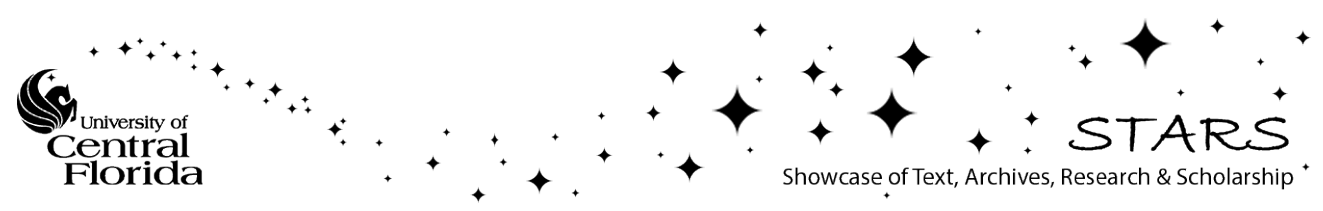




\section{Intensity dependent transmission dynamics in magnetic fluids}

Cite as: Journal of Applied Physics 85, 5953 (1999); https://doi.org/10.1063/1.370002

Published Online: 21 April 1999

Tengda Du, and Weili Luo

\section{ARTICLES YOU MAY BE INTERESTED IN}

Short-time aggregation dynamics of reversible light-induced cluster formation in ferrofluids Journal of Applied Physics 97, 034910 (2005); https://doi.org/10.1063/1.1847706

Nonlinear optical effects in ferrofluids induced by temperature and concentration cross coupling

Applied Physics Letters 72, 272 (1998); https://doi.org/10.1063/1.120710

Thermal lens coupled magneto-optical effect in a ferrofluid

Applied Physics Letters 65, 1844 (1994); https://doi.org/10.1063/1.112861

\section{Lock-in Amplifiers} ... and more, from DC to $600 \mathrm{MHz}$

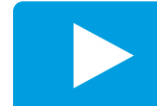

Watch 


\title{
Intensity dependent transmission dynamics in magnetic fluids
}

\author{
Tengda Du and Weili Luo ${ }^{\mathrm{a})}$ \\ Department of Physics, Advanced Material Processing and Analysis Center, Center for Diagnostics \\ and Drug Discovery, University of Central Florida, Orlando, Florida 32826
}

The time dependent transmission is observed when a laser beam passes through a thin layer of a magnetic fluid. We found that the transmission reaches a peak at a time that depends on the intensity of the incident beam - the peak position shifts to shorter time when the input intensity is increased. The peak value of transmitted power decreases with increasing magnetic field. A simplified model is provided to explain these complex dynamics. (C) 1999 American Institute of Physics.

[S0021-8979(99)79608-8]

\section{INTRODUCTION}

The interaction of light with binary liquids has been of growing interest recently due to its broad applications. ${ }^{1-6}$ However, the physical mechanisms associated with many interesting phenomena are still not completely understood. In our previous works, ${ }^{5}$ we reported a new nonlinear optical (NLO) effect in magnetic fluids (MFS) that is attributed to the cross coupling between the temperature and concentration of particles, which in turn leads to the spatial distribution of the refractive index or self-phase modulation. In this article, we report intensity dependent transmission dynamics in a magnetic fluid-we found that there is a maximum in the time dependent transmission and this peak position changes with incident intensity. The mechanism can be explained by a simplified model. We also observed that the peak value of transmitted power decreases while the peak position slightly shifts to longer time in an applied magnetic field below the threshold for field induced instabilities. ${ }^{\text {? }}$

\section{EXPERIMENTAL SETUP}

The sample used in this study is a magnetic fluid consisting of magnetite particles (volume fraction 6\%) suspended in kerosene. Each particle has a diameter of $9 \mathrm{~nm}$ and is coated with a surfactant layer of $2 \mathrm{~nm}$ in thickness. The absorption coefficient of the magnetic fluid, $\sigma$, is measured to be $550 \mathrm{~cm}^{-1}$. The sample is confined between two parallel glass plates separated by a spacer with a thickness of 100 $\mu \mathrm{m}$. Figure 1 illustrates the schematic of the experiment setup. A He-Ne laser $(\lambda=633 \mathrm{~nm})$ with a maximum output of $40 \mathrm{~mW}$ in a single Gaussian mode is used. The laser beam is focused from an original diameter of $1.2 \mathrm{~mm}$ to smaller sizes on the sample by different lenses. The output power is detected by a photodiode connecting to a computer. The magnetic field is applied normally to the sample and parallel to the laser beam.

\footnotetext{
${ }^{a)}$ Electronic mail: 1uo@pegasus.cc.ucf.edu
}

\section{RESULTS AND DISCUSSIONS}

We measure the output power transmitted from the sample, $P_{\text {out }}$, versus time when the laser beam is abruptly switched on. In Fig. 2 we plot time dependence of $P_{\text {out }}$ for input laser power $P_{\text {in }}=6.6 \mathrm{~mW}$ and for focal length $f=20$, $25,40,60,80$, and $100 \mathrm{~mm}$, respectively. From the relation between the focal length, $f$, and the beam width $w, w$ $=\lambda f / \pi w_{0}\left(w_{0}\right.$ is the original beam radius), we know that the longer the focal length $f$, the smaller the intensity because the intensity follows the relation $I_{0}=P_{i n} / \pi w^{2}, I(r)=I_{0} \exp$ $\left(-r^{2} / w^{2}\right)$. Results in Fig. 2 show that the peak position shifts towards longer time with decreased intensity. The time dependence indicates that, before the peaks appear, the magnetic particles move away from the optical axis therefore the sample becomes more transparent, thus $P_{\text {out }}$ increases. The decrease of $P_{\text {out }}$, after reaching the maximum, is corresponding to the reverse process. The concentration change obeys thermal conduction and mass conservation equations ${ }^{8,9}$

$$
\frac{\partial T}{\partial t}-\chi \Delta T=\frac{\sigma I}{\rho C_{p}},
$$

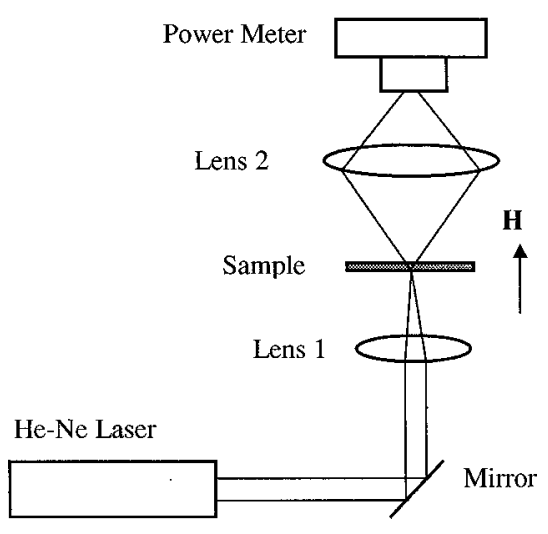

FIG. 1. The schematic of experimental setup. 


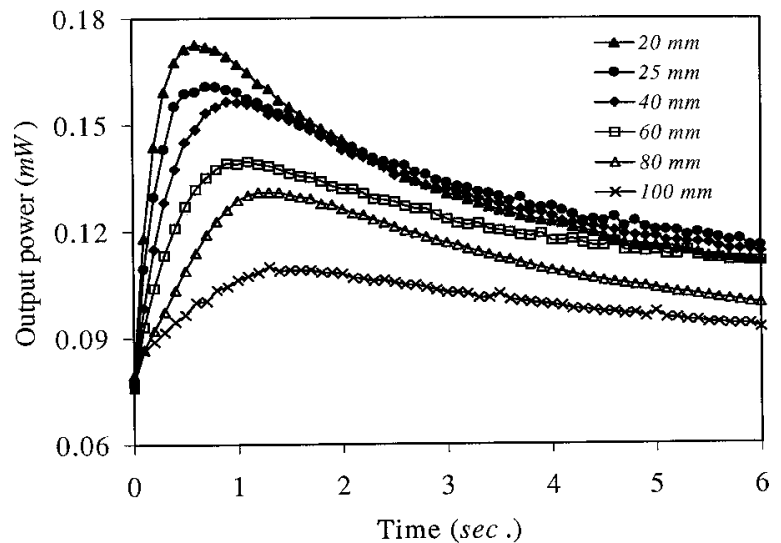

FIG. 2. The transmitted power from the sample vs time in zero field with different focusing lenses.

$$
\frac{\partial c}{\partial t}=D \Delta c+D_{T} \nabla \cdot[c(1-c) \nabla T],
$$

where $I(r, z)=I_{0} \exp \left(-r^{2} / w^{2}-\sigma z\right), T$ is temperature, $c$ the particle concentration, $I$ the intensity of laser beam, $w$ the laser beam width, $\rho$ the density of the fluid, $C_{p}$ the heat capacity, and $\chi$ the thermal diffusivity. $D$ and $D_{T}$ are mass and thermal diffusion coefficients, respectively. For low concentration we assume the absorption coefficient, $\sigma$, is proportional to concentration $c$, or $\sigma=\alpha c, \alpha$ is a constant, so Eqs. (1) and (2) are coupled. To solve the coupled differential equations is not easy, but we can qualitatively describe the temporal behavior at a fixed location, assuming that the beam shape always follows a Gaussian profile.

We rewrite $c=c_{0}\left(1+c_{1}\right)$ where $c_{0}$ is concentration at room temperature, $c_{1}$ is the relative change of concentration; Eq. (2) becomes

$$
\frac{\partial c_{1}}{\partial t}=D \Delta c_{1}+D_{T} \Delta T+D_{T} \nabla T \cdot \nabla c_{1},
$$

where we assume the change of concentration $c_{1} \ll 1$ for low $c$. It is reasonable to choose a location where changes of $T$ and $c$ reach extremes (such on optical axis and the first interface at the glass plates). We further assume that the temperature and concentration in radial direction follow the distribution of the intensity, i.e., the Gaussian, the only difference compared with the beam profile outside the sample is that the beam has a wider distribution width: $w_{1}$ $=k w$, with $k>1$. We also assume that the temperature change in the sample follows a parabolic distribution in $z$ direction with a maximum at $z=0$, where the laser beam enters the sample, and vanishes at $z=L$, where the beam exits the system, assuming that the glass plates have a perfect conductivity. The cross term in Eq. (3) is zero at this reference point $(r=0, z=0)$. We obtain

$$
\begin{aligned}
& \Delta c_{1}(r=0) \approx-c_{1}(r=0)\left(\frac{4}{w_{1}^{2}}+\frac{2}{L^{2}}\right), \\
& \Delta T(r=0) \approx-T(r=0)\left(\frac{4}{w_{1}^{2}}+\frac{2}{L^{2}}\right),
\end{aligned}
$$

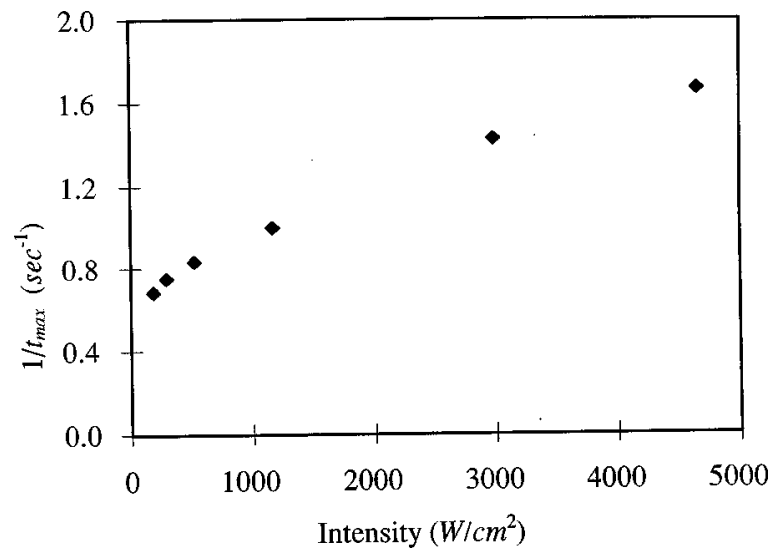

FIG. 3. The term $1 / t_{\max }$ as a function of incident intensity $I_{0}$, where $t_{\max }$ is the peak position.

where $L$ is the thickness of the sample. Substitute $\Delta c_{1}$ and $\Delta T$ into Eqs. (1) and (3), and eliminate one parameter, for example $T$, and we finally get

$$
\frac{\partial^{2} c_{1}}{\partial t^{2}}+\frac{\chi+D}{R^{2}} \frac{\partial c_{1}}{\partial t}+\left(\frac{D \chi}{R^{4}}+\frac{\sigma D_{T} I_{0}}{R^{2} \rho C_{p}}\right) c_{1}=g\left(I_{0}\right),
$$

where $1 / R^{2}=4 / w_{1}^{2}+2 / L^{2}, g\left(I_{0}\right)$ is a function of intensity. This is exactly a damping oscillation equation. To have an oscillation term, the following condition must be satisfied:

$$
\left(\frac{\chi+D}{R^{2}}\right)^{2}-4\left(\frac{D \chi}{R^{4}}+\frac{\sigma D_{T} I_{0}}{R^{2} \rho C_{p}}\right)<0 .
$$

The oscillation frequency is equal to

$$
\omega=\sqrt{\frac{D \chi}{R^{4}}+\frac{\sigma D_{T} I_{0}}{R^{2} \rho C_{p}}-\left(\frac{\chi+D}{2 R^{2}}\right)^{2}} .
$$

The peak position $t_{\max }$ in experiments should be proportional to $1 / \omega$, if we assume the dynamics of total transmission follows the concentration change at the reference point $(r=0$, $z=0$ ) (the distribution shape of $c$ does not change with time). In our system, $D / \chi \sim 10^{-3}$. The above expression can be further simplified as

$$
\omega=\frac{1}{2 R^{2}} \sqrt{\frac{4 \sigma D_{T} I_{0} R^{2}}{\rho C_{p}}-\chi^{2}} .
$$

Substituting the relation between $w_{1}$ and $I_{0}$, finally we have

$$
\omega=\left(\frac{2 \pi I_{0}}{P_{\text {in }}}+\frac{1}{L^{2}}\right) \sqrt{\frac{8 \sigma D_{T} I_{0}}{\rho C_{p}} /\left(\frac{2 \pi I_{0}}{P_{\text {in }}}+\frac{1}{L^{2}}\right)-\chi^{2}} .
$$

Here we take $k=1$ or $w_{1}=w$ for simplicity. Let us look at two extreme cases

(1) $w_{1} \gg L$, or lower intensity, then: $1 / t_{\max }^{2}=a I_{0}-b$,

where $a$ and $b$ are constants.

(2) $w_{1} \ll L$, or higher intensity, then: $1 / t_{\max } \propto I_{0}$.

This relation is qualitatively confirmed by the experimental results. In Fig. 3 we plot $1 / t_{\max }$ as a function of intensity using experimental results. It reasonably agrees with the above qualitative analysis.

Besides the thermal diffusion and mass diffusion that are important mechanisms responsible for redistribution of particles under the influence of laser beam, other factors such as 


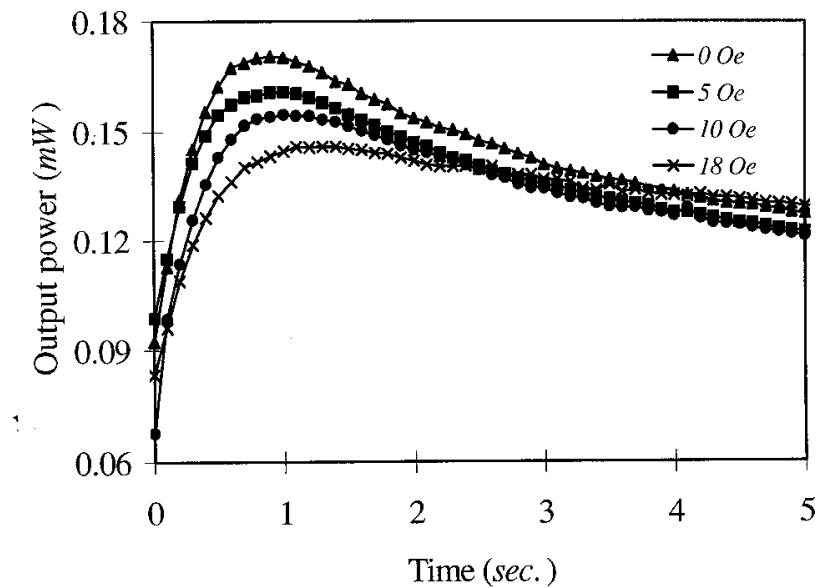

FIG. 4. The transmitted power vs time in different applied magnetic fields with focal length $f=20 \mathrm{~mm}$.

radiation pressure, which includes gradient force (proportional to the gradient of intensity), scattering force, and absorption force, ${ }^{10}$ should be considered as well. For the intensity range we used, these effects are not key factors.

Now we consider the effect of magnetic field on the complex dynamics. Figure 4 shows output powers versus time for different fields. The peak position slightly shifts, but more importantly, the peak value decreases when magnetic field increases from 0 to 18 Oe. This suppression of the transmission might be due to a magnetic body force that results from the spatial distribution of concentration and temperature, namely, the so-called Kelvin force. The force density can be expressed as ${ }^{7}$

$$
f=\frac{\mu_{0} \chi_{m}^{2} H^{2}}{\left(1+\chi_{m}\right)^{3}}\left(\frac{\nabla T}{T}-\frac{\nabla c}{c}\right),
$$

where $H$ is the external magnetic field, $\chi_{m}$ is the susceptibility of the magnetic fluid. Because both $\nabla T$ and $-\nabla c$ are toward the center of the system, the Kelvin force is centripetal. The pressure from this force may resist thermal diffusion when the laser is switched on. To completely understand the effect of Kelvin force on transmission we need to solve several coupled differential equations. Because analytical solutions to these coupled equations are unlikely, numerical calculations will be studied in the near future.

\section{CONCLUSION}

Complex behaviors are observed in the dynamics of transmission from a magnetic fluid. We found that the transmission reaches a peak position that depends on incident intensity. The observations are explained by the cross coupling between the temperature and particle concentration of the system via thermal diffusion. The intensity dependence indicates a nonlinear optical behavior that originates from above novel mechanism. The influence of magnetic field on the transmission is also discussed. Kelvin body force might play an important role. These results will have potential applications in heat and mass transfers and optical devices.

\section{ACKNOWLEDGMENTS}

The authors are grateful to Dr. R. E. Rosensweig for providing the sample. This work is supported by the National Science Foundation Young Investigator Award (Luo).

${ }^{1}$ T. Du, S. Yuan, and W. Luo, Appl. Phys. Lett. 65, 1844 (1994).

${ }^{2}$ M. Kreuzer, T. Tschudi, W. H. de Jeu, and R. Eidenschink, Appl. Phys. Lett. 62, 1712 (1993).

${ }^{3}$ T. Du and W. Luo, Mod. Phys. Lett. B 9, 1643 (1995).

${ }^{4}$ P. W. Smith, P. J. Maloney, and A. Ashkin, Opt. Lett. 7, 347 (1982).

${ }^{5}$ T. Du and W. Luo, Appl. Phys. Lett. 72, 272 (1998).

${ }^{6}$ N. V. Tabiryan and W. Luo, Phys. Rev. E 57, 4431 (1998).

${ }^{7}$ W. Luo, T. Du, and J. Huang (unpublished).

${ }^{8}$ K. E. Grew and T. L. Ibbs, Thermal Diffusion in Gases (Cambridge University Press, Cambridge, 1952), p. 107.

${ }^{9}$ L. D. Landau and E. M. Lifshiz, Fluid Mechanics (Pergamon, Oxford, 1987), p. 192.

${ }^{10}$ K. Svoboda and S. M. Block, Opt. Lett. 19, 930 (1994). 